\title{
Vertical Ge-Si Nanowires with Suspended Graphene Top Contacts as Dynamically- tunable Multispectral Photodetectors
}

\author{
Shi Qiang. Li ${ }^{1 \#}$, Amit Solanki ${ }^{2}$, Jacopo Frigerio ${ }^{3}$, Daniel Chrastina ${ }^{3}$, Giovanni Isella ${ }^{3}$, Changxi \\ Zheng $^{4,5}$, Arman Ahnood ${ }^{6}$, Kumaravelu Ganesan ${ }^{6}$, and Kenneth B. Crozier ${ }^{1,6, *}$ \\ ${ }^{1}$ Department of Electrical and Electronic Engineering, University of Melbourne, Victoria 3010, Australia \\ ${ }^{2}$ School of Engineering and Applied Science, Harvard University, 33 Oxford Street, Cambridge, Massachusetts \\ 02138, United States \\ ${ }^{3}$ Dipartimento di Fisica del Politecnico di Milano, L-NESS, Polo Regionale di Como, Via Anzani 42, I-22100 \\ Como, Italy \\ ${ }^{4}$ School of Physics and Astronomy, Monash University, Clayton, Victoria 3800, Australia \\ ${ }^{5}$ ARC Centre of Excellence in Future Low-Energy Electronics Technologies, Monash University, Clayton, Victoria \\ 3800, Australia \\ ${ }^{6}$ School of Physics, University of Melbourne, Victoria 3010, Australia \\ *Corresponding author: Email kenneth.crozier@unimelb.edu.au \\ ${ }^{\#}$ Current address: Institute of Materials Research and Engineering (IMRE), A*STAR, 2 Fusionopolis Way, \\ Singapore 138634
}

\begin{abstract}
Numerous applications would be enabled by pixels for multispectral imaging whose spectral responses can be dynamically tuned and that can be potentially manufactured at low cost. Here, we show such a capability, by experimentally demonstrating arrays of vertically-oriented germanium-silicon heterojunction nanowires with graphene top contacts. Our devices present opportunities for multispectral imaging because their responsivity spectra can be tailored by choice of nanowire radius for enhanced absorption at certain wavelengths across the visible to short-wave infrared. Importantly, these responsivity spectra can also be dynamically tuned by bias voltage. We demonstrate this experimentally by tuning the responsivity peak of a single pixel across the visible region by varying the bias voltage and by showing that this would allow red/green/blue channels to be reconstructed. This opens the exciting prospect of a single pixel that can resolve color (i.e. replacing the three red/green/blue pixels of traditional approaches) or even resolve several bands for multispectral imaging.
\end{abstract}

Keywords: Multispectral photodetector; Nanowires; Surface States; Germanium Epitaxy 
The rise of the internet-of-things, mobile computing, mobile communication technologies, and robotics (machine vision) is producing a surging demand for sensors with low cost, reduced form factor, high performance, enhanced functionality, and wide spectral response. Therefore, recent years have seen a trend toward image sensors with ever-smaller pixels due to tremendous market demand for small cameras with high pixel counts. At the time of writing, image sensors have been produced whose small pixel size (sub-micron) makes cross-talk between adjacent pixels inevitable ${ }^{1}$, partly due to the limitations of traditional color filters based on absorptive dyes. Filterless image sensor pixels based on nanowires ${ }^{2}$ have emerged as a promising alternative technology. In such devices, separation of different spectral channels is enabled by the fact that the spectral responsivities of the nanowires can engineered by appropriate choice of their radii, making filters not needed. In the fabrication process, the spectral responsivities of multiple devices with different properties can be defined in a single lithographic step, which is advantageous for simplifying the manufacturing process.

In addition to the nanowire approach, filter-less photodetectors achieving wavelength selectivity have been demonstrated using organic and perovskite materials. ${ }^{3-6}$ In these demonstrations, the active media were purely organic materials, ${ }^{4,6}$ purely perovskite materials, ${ }^{5}$ or a combination of organic and perovskite materials. ${ }^{3}$ In some of these studies, surface defects played a central role to the wavelength-selective photoresponse. ${ }^{3-5}$ Organic and perovskite materials, however, often face stability challenges that could pose an obstacle to the commercialization of these devices ${ }^{7-8}$ Wavelength-selective filter-less photodetectors produced in standard materials platforms are thus desired, but the nanowire devices we previously demonstrated were silicon ${ }^{2,9}$ and thus unsuitable for many applications in multispectral imaging for which longer wavelength response is needed. We recently demonstrated spectrally-selective germanium nanowire photodetectors in the visible to short-wave infrared (SWIR). ${ }^{10}$ These nanowires were on germanium substrates however. The germanium-on-silicon platform on the other hand presents interesting opportunities as a platform for multispectral imaging devices. It is compatible with current silicon technology, thereby substantially reducing its cost compared to the use of germanium substrates. Photodetectors based on germanium-on-silicon have been furthermore demonstrated with high speed response ( $>30$ $\mathrm{GHz})^{11-14}$ Spectrally-selective photodetection with germanium-on-silicon nanowires has not been previously demonstrated however. The use of nanowires furthermore allow us to take advantage of the advances that have been made in the fabrication and device physics of photodetectors based on nanowires ${ }^{15-20}$ and related structures ${ }^{21-22}$.

Here we demonstrate a new type of multispectral imaging pixel that comprises vertical germanium-silicon heterojunction nanowires with a suspended graphene top electrode. The pixels are spectrally-selective, i.e. have responsivity spectra exhibiting peaks whose positions are controlled by appropriate choice of nanowire radius. We furthermore demonstrate that the responsivity spectrum of a single pixel can be tuned dynamically by voltage during operation. This capability originates from a combined effect of the nanowire's photonic resonances and surface states. The dynamic tuning capability could potentially reduce the pixels needed for color imaging from three to one, or even be used for multispectral imaging with a single pixel. Furthermore, we note that our pixels cover a wide spectral range that extends from ultra-violet $(350 \mathrm{~nm})$ to shortwave infrared $(1500 \mathrm{~nm})$. 


\section{Device Fabrication}

Fabrication of our device starts with a silicon wafer (100), doped $n+$ with arsenic (resistivity: 0.001 to $0.01 \mathrm{Ohm}-\mathrm{cm})$. Thin films of germanium $(\mathrm{Ge})$ are then grown epitaxially. The first layer is intrinsic Ge, with a thickness of $1000 \mathrm{~nm}$. This serves as the active layer of our device, from which photogenerated carriers are collected. The next (top) layer is $\mathrm{p}+\mathrm{Ge}$, doped with boron at $5 \times 10^{18}$ $\mathrm{cm}^{-3}$. This yields the structure shown schematically as Fig. 1a. The wafer is cleaned in hydrofluoric acid (1\%), and titanium (5 nm, for adhesion) and gold (100 nm) films are evaporated on the backside. The wafer is then covered with photoresist and diced into $14 \mathrm{~mm}$ by $14 \mathrm{~mm}$ chips for subsequent processing.

We next remove the photoresist by soaking the chip in acetone and rinsing it in isopropanol for one minute. Electron-beam lithography resist (ZEP520A, Zeon Corporation) is then spin-coated onto the chip to a thickness of $150 \mathrm{~nm}$. The chip is then baked on a hotplate for 3 minutes (at 180 ${ }^{\circ} \mathrm{C}$ ). Following that, the chip is patterned by electron-beam lithography (EBPG5000plusES, Vistec), with a dose of $200 \mu \mathrm{C} / \mathrm{cm}^{2}$. The exposed chip is then developed (ZED-N50, n-Amyl acetate) for 60 seconds and rinsed.

A stack of metals is then deposited on the chip by electron beam evaporation. In order of deposition, the stack comprises $\mathrm{Ti} / \mathrm{Au} / \mathrm{Ni} / \mathrm{Al}$ at thicknesses of $10 \mathrm{~nm} / 30 \mathrm{~nm} / 20 \mathrm{~nm} / 10 \mathrm{~nm}$, respectively. Each metal layer has a specific purpose. Aluminum is the etch mask, for the subsequent reactive ion etching step to form the nanowires. The nickel layer serves to block diffusion between the gold and aluminum layers. The purpose of the gold layer is to provide good electrical contact to the graphene monolayer that is later transferred to the chip and serves as a top electrode. The titanium layer serves to promote adhesion with the Ge substrate.

After metal deposition, the lift-off process is performed, resulting in a two-dimensional array of metal disks on the chip (Fig. 1b). These arrays are generally chosen to have extents of $200 \times 200 \mu \mathrm{m}$, and the disks range in radii from $75 \mathrm{~nm}$ to $150 \mathrm{~nm}$. The disks are in square arrays with periods of $800 \mathrm{~nm}$.

Inductively-coupled plasma reactive ion etching (ICP-RIE, Oxford Plasmalab 100) is then performed on the chip, resulting in arrays of nanowires with vertical profiles (Fig. 1c). A modified Bosch process is used. In this process, instead of cycling between a deposition process with $\mathrm{C}_{4} \mathrm{~F}_{8}$ gas and an etching process with $\mathrm{SF}_{6}$ gas, the two gases are simultaneously introduced into the RIE chamber. $^{10,23}$ The nanowires have heights of $1500 \mathrm{~nm}$, with the top part $(1200 \mathrm{~nm})$ being germanium and the bottom part $(300 \mathrm{~nm})$ being silicon. The aluminum mask is then removed by ICP-RIE.

Immediately after the etching, the chip is transferred to a plasma-enhanced chemical vapor deposition (PECVD) system (Oxford Plasmalab 100), and silicon dioxide is deposited to a thickness of $1000 \mathrm{~nm}$ (Fig. 1d). Photoresist is then spun onto the chip, and photolithography is performed to define a circular opening (100 $\mu \mathrm{m}$ diameter) that is centered over the nanowire array. ICP-RIE is then performed to etch the silicon dioxide in a directional fashion. A modified Bosch process is again used, with $\mathrm{C}_{4} \mathrm{~F}_{8}$ and $\mathrm{SF}_{6}$ introduced simultaneously ${ }^{10,23}$ This leads to the silicon dioxide being removed from the tops of the nanowires (exposing the germanium). In between the nanowires, the silicon dioxide is etched down to the flat silicon surface. By appropriate choice of the process parameters used in the etching step, a silicon dioxide coating can be left on each 
nanowire or completely removed (as depicted in Fig. 1e). As we discuss later, this layer provides electrical passivation of the nanowire surface.

We next deposit gold to a thickness of $4 \mathrm{~nm}$ by electron-beam evaporation (at rate of $2 \dot{A} / s$ ) onto a piece of copper foil covered by a monolayer of graphene grown by chemical vapor deposition (CVD). This substrate with graphene is procured from Graphenea Inc. The graphene monolayer (with gold coating on top) is then transferred to the Ge-Si nanowire chip via wet transfer. The gold coating on the graphene has two functions. First, it enhances the conductivity. Second, it provides mechanical support that helps the graphene transfer process. After transfer, the suspended graphene sheet extends out from the active region (where it contacts the tops of the nanowires) to the silicon dioxide platform (Fig. 1f).

We now consider our choice for the top electrode (graphene with thin gold layer) by comparing it with two alternatives. We perform this comparison by simulating external quantum efficiency (EQE) spectra for various configurations. In these calculations, the EQE is taken as the fraction of incident light absorbed in the nanowire intrinsic regions. This is an appropriate assumption for these calculations, whose purpose is to compare different choices for the top electrode. In these simulations (Fig. 1g), the nanowires have radii of $90 \mathrm{~nm}$, and the $\mathrm{SiO}_{2}$ coating is $80 \mathrm{~nm}$ thick. For the graphene-only case (blue curve), it can be seen that the peak EQE exceeds $90 \%$. As discussed, we previously demonstrated photodetectors consisting of arrays of vertical silicon nanowires ${ }^{2}$. In that work, the spaces between the nanowires were filled with poly-methyl methacrylate (PMMA), to provide mechanical support to the top electrode, the transparent conductor indium tin oxide (ITO). From Fig. 1g (red curve), however, it can be seen that filling the spaces between the nanowires with PMMA reduces the EQE (in comparison to the graphene-only case). This is a consequence of the reduced refractive index contrast between the nanowire and its surroundings. It would also be difficult to extend this device concept to the ultraviolet (UV) with the PMMA filling layer due to absorption, which is detrimental because it reduces the optical transmission and affects long-term stability. Furthermore, at longer wavelengths (e.g. 800-2000 nm), PMMA has significant absorption peaks from the vibrational overtones of $\mathrm{C}-\mathrm{H}$ bonds, which limits optical transmission. ${ }^{24}$ Graphene on the other hand does not face these challenges, is mechanically strong, ${ }^{25}$ and is stable under intense UV irradiation for prolonged periods. ${ }^{26}$

To facilitate the process of transferring the graphene to the nanowire chip, increasing its thickness with an additional layer is in general helpful. PMMA is commonly chosen as this additional layer but would be difficult to remove after transfer. From simulations (data not shown here), we also find that when the top electrode consists of graphene with a PMMA film, the device's EQE curve exhibits oscillations. For a top electrode consisting of graphene with a thin gold layer, the EQE spectrum (orange curve of Fig. 1g) shows some oscillations, but these are not large. We thus choose gold-covered graphene as the top electrode because it is practical from a fabrication standpoint, does not have pronounced Fabry-Perot oscillations, is stable in the wavelength range of interest, and has competitive EQE. It furthermore offers good electrical conductivity.

\section{Passivation by silicon dioxide}

Surfaces play significant roles in nanostructures, due to their inherently high surface-to-volume ratios. Indeed, surfaces can change the nature of ${ }^{27}$, amplify ${ }^{28}$ or diminish ${ }^{29}$ properties observed in bulk materials. For nanowire devices such as metal-oxide-semiconductor field-effect transistors

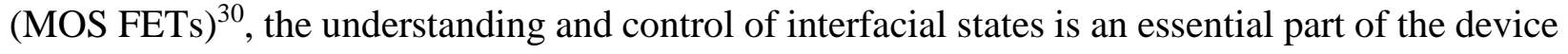


design process. The consideration of interfacial states is particularly important for our devices because the oxidation of germanium does not help to passivate its surface, ${ }^{31-32}$ unlike the situation for silicon, whose native oxide is intrinsically stable and provides excellent passivation. This motivates us to investigate the use of silicon dioxide to passivate our Ge-Si nanowires.

For comparison, we fabricate nanowire devices with and without a silicon dioxide layer. The unpassivated devices (termed NW PD) are illustrated as Fig. 2a-c, while those passivated with PECVD grown silicon dioxide ( $p$-NW PD) are shown as Fig. 2d-f. It can be seen from the SEM image of Fig. $2 b$ that the etched nanowires are slightly undercut, which is consistent with previous reports on nanowires etched using metal masks. ${ }^{33}$ SEM images of the un-passivated and passivated devices after graphene transfer are shown as Figs 2c and 2f, respectively. Although the spacing between nanowires is quite large, it can be seen that the graphene sits on the tops of the nanowires as a flat sheet, rather than bowing down to the substrate between the nanowires.

We next perform electrical characterization of the fabricated devices by measuring the currentvoltage $(I-V)$ characteristics without illumination (Fig. 3). It can be seen that the I-V characteristic for the $p$-NW PD device (radius $100 \mathrm{~nm}$ ) are distinctly diode-like (rectifying), while the NW PD shows characteristics like that of an Ohmic conductor, although the two devices have the same radius and identical processing parameters other than the passivation. This confirms the importance of surface passivation for these devices. As shown before, ${ }^{34}$ un-passivated germanium nanowires in air can have poor consistency in electrical properties due to the fact that germanium oxide is unstable and easily modified by the ambient environment.

Result for a $p$-NW PD with a nominal radius of $75 \mathrm{~nm}$ is also shown in Fig. 3. It can be seen that the leakage current (reverse bias current) is larger for this device than for that with radius $100 \mathrm{~nm}$. We attribute this to the greater surface-to-volume ratio of the smaller radius nanowires. Charge carriers accumulate at the surface due to band-bending. This effect is more pronounced for the smaller radius nanowires and leads to a steeper reverse current slope.

In the inset of Fig 3., we compare the electrical characteristics of a $p$-NW PD device before and after annealing. We find that annealing in forming gas (hydrogen and nitrogen) at $350{ }^{\circ} \mathrm{C}$ increases the forward current by more than 50 times. We attribute this to improved electrical contact between the metal on the tops of the nanowires and the graphene, as well as further passivation of surface states on the sidewall of the nanowires. ${ }^{35-36}$

\section{Responsivity Measurement Results}

\section{Zero Bias}

We next measure the responsivities of our $p$-NW PD devices, by illuminating them with monochromatic light swept in wavelength from the visible to the short-wave infrared while measuring photocurrent. Details of the experimental set-up are provided in the Supplementary Information (SI). Experimental results for $p$-NW PD devices with radii ranging from 75 to $150 \mathrm{~nm}$ are shown as Fig. 4a. It can be seen that the responsivity spectra exhibit peaks that red-shift with increasing nanowire radius. These arise from interplay between coupling of the incident light to the waveguide modes supported by the nanowires and subsequent absorption of the coupled light, a mechanism that has been discussed in previous work. ${ }^{37}$ Simulated responsivity spectra are shown as Fig. 4b. These are obtained by simulating the fraction of incident light absorbed in the nanowire 
intrinsic region as a function of wavelength. This is then multiplied by $q \lambda /(h c)$ to obtain the responsivity, where $q$ is the electron charge, $\lambda$ is the wavelength, $h$ is Planck's constant and $c$ is the speed of light in vacuum. Further details on the simulation method are provided in the SI. It can be seen that, like the experiments, the simulated responsivity spectra show peaks that shift to longer wavelengths with increasing nanowire radius. To illustrate this further, the simulated responsivity spectra of Fig. 4b are also provided in normalized form in the SI.

While the experiments (Fig. 4a) and simulations (Fig. 4b) show consistent trends, there are some differences that we now discuss. The first difference is that the simulated nanowire devices have smaller radii than their experimental counterparts. It should also be realized that the radii quoted for the latter are nominal values, being the design values used in the electron-beam lithography step. This can be partly attributed to the actual radii of the nanowires being smaller than these nominal radii, due to undercut in the etching process. The presence of the undercut is confirmed by the SEM images (Fig. 2b and Supplementary Figure S-5 of the SI). The second difference is that the simulated devices with different radii have similar values for peak responsivity. The peak values of the responsivity spectra of the actual devices show variation however. We attribute this to fracturing of the graphene layer, which varies from device to device. Efforts to mitigate this are currently underway. Note that this cannot be seen from Fig. 4a, as the curves are all normalized so that each has a peak value of unity. This is necessary to allow comparison of the shapes of the responsivity spectra. The third difference is to do with the widths of the responsivity spectral peaks. The widths of the responsivity peaks measured for $p$-NW-PD devices with nominal radii of 113 $\mathrm{nm}$ and above are in reasonable agreement with their simulated counterparts. The devices with radii of $83 \mathrm{~nm}$ and $100 \mathrm{~nm}$ have measured responsivity peaks that are broader than their simulated counterparts. This is also the case for the device with nominal radius $75 \mathrm{~nm}$. For this device, the experimental measurements also show a responsivity peak that is blue-shifted with respect to the simulations. This is discussed further in the next section. As discussed in the SI, the nanowires exhibit inverted tapering, i.e. their radii vary along the nanowire length, being narrower at the bottom than at the top. For the nanowire with nominal radius $75 \mathrm{~nm}$, for example, the actual radius varies from $50 \mathrm{~nm}$ (at the top) to $45 \mathrm{~nm}$ (at the bottom, Supplementary Figure S-5). As discussed in previous work, ${ }^{38}$ tapering can broaden the spectral peaks. It is possible that the spectral broadening is due to nanowire tapering.

\section{Dynamic Tuning}

Although passivation has been performed, surface states at the interface between silicon dioxide and germanium still exist and can act as traps. The surface area-to-volume ratio increases of course as the nanowire radius is reduced. The thinner nanowires thus have leakage currents that are higher and also vary more strongly under reverse bias, as discussed in previously (Fig. 3). On the other hand, as we discuss further below, surface states present the opportunity for nanowire array devices to have dynamically-tunable spectral response. Surface traps result in an electrical potential being established from the nanowire center to the surface. This potential directs optically generated electrons or holes to the surface and recombination occurs. Photo-excited carriers closer to the surface thus have a higher probability of recombination. However, when a bias is applied, an addition electrical potential is generated along the long axis of the nanowire, orthogonal to the radial direction, subsequently, the charge collection rate increases for the photo-generated electrons and/or holes. 
One might expect this effect to become more significant as the nanowire radius decreases. We thus choose to study this in-depth for the $p$-NW-PD with the smallest radius (nominal value $75 \mathrm{~nm}$, simulated value $35 \mathrm{~nm}$ ). Fig. 5a shows the measured spectral responsivities of this device under different bias voltages from the ultraviolet $(350 \mathrm{~nm})$ to the near-infrared $(900 \mathrm{~nm})$. It can be seen that spectral responsivity changes significantly with bias. The unbiased sample has a responsivity peak at $\lambda \sim 490 \mathrm{~nm}$. When the top electrode is biased negatively at $0.5 \mathrm{~V}$, the peak shifts to $\lambda \sim 600$ $\mathrm{nm}$. After switching the polarity to forward bias $(0.5 \mathrm{~V})$, a distinct peak at $\lambda \sim 390 \mathrm{~nm}$ is observed. This measured behavior is consistent with our optical-electrical model (Fig. 5b) and originates from the strong dependence of the internal quantum efficiency (IQE) spatial map with bias voltage (Fig. 5c). As presented in the SI, this effect is far less pronounced for larger radius nanowires. Before discussing our physical interpretation, however, we discuss a potential application in imaging.

This dynamic tunability of spectral peak across the visible region presents the interesting possibility of a photodetector pixel with a dynamically-tunable response. Such a pixel could replace multiple (red/green/blue) pixels in a color image sensor. The advantages could be even more pronounced for multispectral imaging systems, whose larger numbers of spectral channels often necessitate bulky instrumentation such as rotating filter wheels. To explore this concept, we demonstrate the reconstruction of the $\mathrm{x}, \mathrm{y}$, $\mathrm{z}$ responsivity curves of CIE 1931 standard observer function $^{39}$ as Fig. 5d-f. Each responsivity curve consists of the weighted sum of responsivity spectra measured from the nanowire photodetector pixel with smallest radius (nominal value 75 $\mathrm{nm}$, simulated value $35 \mathrm{~nm}$ ) under eight different bias voltages. The weightings are chosen to minimize the difference between the reconstructed and ideal results. It can be seen from Fig. 5d-f that reconstructed and ideal spectra are in reasonable agreement. It can be expected that these differences would decrease if the number of voltages employed in the reconstruction were to be increased. This might be an interesting topic for future investigations.

We next discuss our physical interpretation of the dynamic tuning properties of our nanowire photodetectors. We first note that measurements from our nanowire devices with slowlymodulated illumination reveal that the photocurrent drifts over time (see Supplementary Information). In these experiments, the illumination is switched at $0.1 \mathrm{~Hz}$ with a $50 \%$ duty cycle, i.e. with on and off times of 5 seconds each. The long decay time observed may be attributed partly to the discharging route being un-optimized, and partly due to slow surface states of the type discussed previously. ${ }^{32}$ These slow surface states originate from slow moving charged ions that respond with a time constant of minutes or longer. To mitigate the influence of the slow states upon our analysis, we modulate the illumination at a rate $(450 \mathrm{~Hz}$, see SI) much higher than the characteristic rates of these slow states. The results of Fig. 4 and 5 are based on this approach.

We attribute the bias-dependent photoresponse to mediation by surface traps. At the semiconductor nanowire surfaces, termination of the crystal lattice inevitably produces mid-gap surface states. ${ }^{40-}$ ${ }^{45}$ For germanium, this leads to an effect known as Fermi level pinning (FLP) that results in the Fermi level being fixed (pinned) at about $0.09 \mathrm{eV}$ above the valence band edge, with what is on the other side of the interface having very little effect. ${ }^{43}$ This energy level is close to the Charge Neutrality Level (CNL), which is the level at which a balance between donor-like trap states and acceptor-like trap states results in charge neutrality. Fermi-level pinning (FLP) causes band bending at the nanowire surface (Fig. 5c, bottom panel). A spatially extended space-charge layer thus exists at the nanowire surface. As we discuss further below, when the device is forward biased, the holes that accumulate at the nanowire surface region enable it to function as a photoconductor. 
In other words, the core of our device can be thought of as acting as a photovoltaic detector, while its surface can be thought of as acting as a photoconductor.

In order to confirm the hypothesis proposed above, we perform a multi-physics simulation in COMSOL Multiphysics, which couples electrical simulations of the semiconductor transport with electromagnetic wave simulation (see the SI for the detailed setup). In the simulations, we implement FLP by fixing the surface Fermi level near the CNL. A good match is obtained between the simulated and experimental responsivities as shown in Fig. 5a and 5b.

We use the same simulation to understand the mechanism of the bias-dependent photoresponsivity by generating a spatial-resolved IQE map of the nanowire, as shown in Fig. 5c. Due to the high surface area-to-volume ratio, the whole nanowire is at the same potential except at the interface between $\mathrm{Si}+$ and germanium region, at which there is a high potential drop. Due to the latter, photogenerated carriers there are readily collected via drift, resulting in high IQE. Reverse bias mainly increases the band-offset around the interface, rather than being evenly distributed across the whole nanowire. This leads to two effects. First, it expands the region with high potential drop. Second, it increases the gradient of the potential drop. These effects lead to changes in the EQE spectrum. As shown in the upper middle panel of Fig. 5c, under reverse bias (500 $\mathrm{mV})$, the high quantum efficiency region at Ge-Si interface becomes longer. This results from the depletion region becoming elongated along the NW axis due to higher potential drop that now occurs, in comparison to the zero-bias case. We next consider what happens under forward bias (500 mV). It can be seen (Fig. 5c, right panel) that the surface has a much higher quantum efficiency than the NW center, and that this efficiency exceeds unity. In other words, the device functions as a photoconductive detector under forward bias. The responsivity spectrum of a nanowire photodetector is determined by the fraction of incident photons that are absorbed and the photocurrent at the device terminals that subsequently results from for example photovoltaic or photoconductive mechanisms. In other words, it is given by the combination of the spatial map of absorption and the spatial map of IQE. For the nanowire considered in Figure 5, it is the control provided by the bias voltage upon the IQE spatial map and thus the responsivity spectrum that allows the device to be dynamically reconfigurable. Lastly, we note that our model is based on the NW only, and does not include the Si substrate. While the responsivity spectra predicted by our model (Fig. 5b) are in reasonable agreement with experiment (Fig. 5a), we cannot rule out the possibility that substrate absorption may also contribute to photocurrent. This might be an interesting topic for future investigation.

\section{Conclusions}

We demonstrate dynamically tunable multispectral pixels based on a hybrid architecture combining 1-D (graphene) and 2-D (nanowire) materials. The latter are based on materials (silicon and germanium) that have been used in a combined fashion for decades in the semiconductor industry. We anticipate that the ability to tailor responsivity spectra from visible to short-wave infrared wavelengths would be advantageous for multispectral imaging applications. We show that this can be achieved in both static (i.e. via nanowire radius) and dynamic (i.e. via bias voltage) modes of operation. We lastly note that while the continuing trend toward smaller and smaller pixels is largely motivated by consumer demand for image sensors with ever increasing pixel counts, this work reveals that other phenomena occurring at sub-wavelength scales have potential applications. 


\section{Acknowledgements}

This work was supported by the Australian Research Council (DP150103736, DP180104141, and FT140100577) and by VESKI. Electron microscopy was performed at the Bio21 Advanced Microscopy Facility, the University of Melbourne. Fabrication was performed in part at the Melbourne Centre for Nanofabrication (MCN) in the Victorian Node of the Australian National Fabrication Facility (ANFF).

\section{Associated Content}

Supporting Information Available. We have provided a SI document featuring the detailed supporting information, such as

- Setup for photo-responsivity measurements;

- On-off switching experiment;

- Additional SEM and TEM images of the nanowires;

- Transmission of Gr/Au composite;

- Details on germanium epitaxial growth;

- Details on optical and electrical simulations;

- Details on color reconstruction with biased photodetector.

This material is available free of charge via the Internet at http://pubs.acs.org

\section{References}

(1) Taguchi, H.; Enokido, M. In Technology of color filter materials for image sensor, Proc. Int. Image Sensor Workshop, 2011; pp 8-11.

(2) Park, H.; Dan, Y.; Seo, K.; Yu, Y. J.; Duane, P. K.; Wober, M.; Crozier, K. B., Filter-free image sensor pixels comprising silicon nanowires with selective color absorption. Nano Lett. 2014, 14, 1804-1809.

(3) Lin, Q.; Armin, A.; Burn, P. L.; Meredith, P., Filterless narrowband visible photodetectors. Nat. Photonics 2015, 687-694.

(4) Armin, A.; Jansen-van Vuuren, R. D.; Kopidakis, N.; Burn, P. L.; Meredith, P., Narrowband light detection via internal quantum efficiency manipulation of organic photodiodes. Nat. Commun. 2015, 6, 6343.

(5) Fang, Y.; Dong, Q.; Shao, Y.; Yuan, Y.; Huang, J., Highly narrowband perovskite singlecrystal photodetectors enabled by surface-charge recombination. Nat. Photonics 2015, 9, 679-686.

(6) Wang, W.; Zhang, F.; Du, M.; Li, L.; Zhang, M.; Wang, K.; Wang, Y.; Hu, B.; Fang, Y.; Huang, J., Highly Narrowband Photomultiplication Type Organic Photodetectors. Nano Lett. 2017, 17, 1995-2002.

(7) Yang, Y.; You, J., Make perovskite solar cells stable. Nature 2017, 544, 155-156.

(8) Cheng, P.; Zhan, X., Stability of organic solar cells: challenges and strategies. Chem. Soc. Rev. 2016, 45, 2544-2582.

(9) Park, H.; Crozier, K. B., Vertically stacked photodetector devices containing silicon nanowires with engineered absorption spectra. ACS Photonics 2015, 2, 544-549. 
(10) Solanki, A.; Li, S.; Park, H.; Crozier, K. B., Harnessing the interplay between photonic resonances and carrier extraction for narrowband germanium nanowire photodetectors spanning the visible to infrared. ACS Photonics 2017, 5, 520-527.

(11) Liu, J.; Michel, J.; Giziewicz, W.; Pan, D.; Wada, K.; Cannon, D. D.; Jongthammanurak, S.; Danielson, D. T.; Kimerling, L. C.; Chen, J., High-performance, tensile-strained Ge pin photodetectors on a Si platform. Appl. Phys. Lett. 2005, 87, 103501.

(12) Colace, L.; Masini, G.; Assanto, G.; Luan, H.-C.; Wada, K.; Kimerling, L., Efficient highspeed near-infrared Ge photodetectors integrated on Si substrates. Appl. Phys. Lett. 2000, 76, 1231-1233.

(13) Michel, J.; Liu, J.; Kimerling, L. C., High-performance Ge-on-Si photodetectors. Nat. Photonics 2010, 4, 527-534.

(14) Vivien, L.; Rouvière, M.; Fédéli, J.-M.; Marris-Morini, D.; Damlencourt, J.-F.; Mangeney, J.; Crozat, P.; El Melhaoui, L.; Cassan, E.; Le Roux, X., High speed and high responsivity germanium photodetector integrated in a Silicon-On-Insulator microwaveguide. Opt. Express 2007, 15, 9843-9848.

(15) Yang, C.; Barrelet, C. J.; Capasso, F.; Lieber, C. M., Single p-type/intrinsic/n-type silicon nanowires as nanoscale avalanche photodetectors. Nano Lett. 2006, 6, 2929-2934.

(16) Wang, J.; Gudiksen, M. S.; Duan, X.; Cui, Y.; Lieber, C. M., Highly polarized photoluminescence and photodetection from single indium phosphide nanowires. Science 2001, 293, 1455-1457.

(17) Hayden, O.; Agarwal, R.; Lieber, C. M., Nanoscale avalanche photodiodes for highly sensitive and spatially resolved photon detection. Nat. Mater. 2006, 5, 352-356.

(18) Tian, B.; Zheng, X.; Kempa, T. J.; Fang, Y.; Yu, N.; Yu, G.; Huang, J.; Lieber, C. M., Coaxial silicon nanowires as solar cells and nanoelectronic power sources. Nature 2007, 449, 885889.

(19) Wallentin, J.; Anttu, N.; Asoli, D.; Huffman, M.; Åberg, I.; Magnusson, M. H.; Siefer, G.; Fuss-Kailuweit, P.; Dimroth, F.; Witzigmann, B., InP nanowire array solar cells achieving 13.8\% efficiency by exceeding the ray optics limit. Science 2013, 339, 1057-1060.

(20) Cao, L.; White, J. S.; Park, J.-S.; Schuller, J. A.; Clemens, B. M.; Brongersma, M. L., Engineering light absorption in semiconductor nanowire devices. Nat. Mater. 2009, 8, 643647.

(21) Juntunen, M. A.; Heinonen, J.; Vähänissi, V.; Repo, P.; Valluru, D.; Savin, H., Near-unity quantum efficiency of broadband black silicon photodiodes with an induced junction. Nat. Photonics 2016, 10, 777.

(22) Cao, L.; Park, J.-S.; Fan, P.; Clemens, B.; Brongersma, M. L., Resonant germanium nanoantenna photodetectors. Nano Lett. 2010, 10, 1229-1233.

(23) Saffih, F.; Con, C.; Alshammari, A.; Yavuz, M.; Cui, B., Fabrication of silicon nanostructures with large taper angle by reactive ion etching. JVST B 2014, 32, 06 FI04. (24) Eldada, L.; Blomquist, R.; Shacklette, L. W.; McFarland, M. J., High-performance polymeric componentry for telecom and datacom applications. Opt. Eng. 2000, 39, 596-609. (25) Lee, C.; Wei, X.; Kysar, J. W.; Hone, J., Measurement of the elastic properties and intrinsic strength of monolayer graphene. Science 2008, 321, 385-388.

(26) Das, S. R.; Nian, Q.; Saei, M.; Jin, S.; Back, D.; Kumar, P.; Janes, D. B.; Alam, M. A.; Cheng, G. J., Single-layer graphene as a barrier layer for intense UV laser-induced damages for silver nanowire network. ACS Nano 2015, 9, 11121-11133. 
(27) Shalish, I.; Temkin, H.; Narayanamurti, V., Size-dependent surface luminescence in ZnO nanowires. Phys. Rev. B 2004, 69, 245401.

(28) He, R.; Yang, P., Giant piezoresistance effect in silicon nanowires. Nat. Nanotechnol. 2006, 1, 42-46.

(29) Kayes, B. M.; Atwater, H. A.; Lewis, N. S., Comparison of the device physics principles of planar and radial pn junction nanorod solar cells. J. Appl. Phys. 2005, 97, 114302.

(30) Sze, S. M.; Ng, K. K., Physics of semiconductor devices. John wiley \& sons: 2006.

(31) Many, A.; Gerlich, D., Distribution and cross sections of fast states on germanium surfaces in different gaseous ambients. Phys. Rev. 1957, 107, 404.

(32) Kingston, R., Review of germanium surface phenomena. J. Appl. Phys. 1956, 27, 101114.

(33) Henry, M. D.; Walavalkar, S.; Homyk, A.; Scherer, A., Alumina etch masks for fabrication of high-aspect-ratio silicon micropillars and nanopillars. Nanotechnology 2009, $20,255305$.

(34) Li, Y.; Clady, R.; Marshall, A. F.; Park, J.; Thombare, S. V.; Chan, G.; Schmidt, T. W.; Brongersma, M. L.; McIntyre, P. C., Ultrafast Carrier Dynamics of a Photo-Excited Germanium Nanowire-Air Metamaterial. ACS Photonics 2015, 2, 1091-1098.

(35) Lee, J. M.; Choung, J. W.; Yi, J.; Lee, D. H.; Samal, M.; Yi, D. K.; Lee, C.-H.; Yi, G.-C.; Paik, U.; Rogers, J. A., Vertical pillar-superlattice array and graphene hybrid light emitting diodes. Nano Lett. 2010, 10, 2783-2788.

(36) Minaye Hashemi, F. S.; Thombare, S.; Morral, A. F. i.; Brongersma, M. L.; McIntyre, P. C., Effects of surface oxide formation on germanium nanowire band-edge photoluminescence. Appl. Phys. Lett. 2013, 102, 251122.

(37) Seo, K.; Wober, M.; Steinvurzel, P.; Schonbrun, E.; Dan, Y.; Ellenbogen, T.; Crozier, K. B., Multicolored vertical silicon nanowires. Nano Lett. 2011, 11, 1851-1856.

(38) Solanki, A.; Crozier, K., Vertical germanium nanowires as spectrally-selective absorbers across the visible-to-infrared. Appl. Phys. Lett. 2014, 105, 191115.

(39) Fairchild, M. D., Color appearance models. John Wiley \& Sons: 2013.

(40) Shockley, W., On the surface states associated with a periodic potential. Phys. Rev. 1939, 56, 317.

(41) Bardeen, J., Surface states and rectification at a metal semi-conductor contact. Phys. Rev. 1947, 71, 717.

(42) Tamm, I., On the possible bound states of electrons on a crystal surface. Phys. Z. Sov. Un. 1932, 1, 733.

(43) Dimoulas, A.; Tsipas, P.; Sotiropoulos, A.; Evangelou, E., Fermi-level pinning and charge neutrality level in germanium. Appl. Phys. Lett. 2006, 89, 252110.

(44) Goodwin, E. In Electronic states at the surfaces of crystals, Mathematical Proceedings of the Cambridge Philosophical Society, Cambridge Univ Press: 1939; pp 205-220.

(45) Garnett, E. C.; Tseng, Y.-C.; Khanal, D. R.; Wu, J.; Bokor, J.; Yang, P., Dopant profiling and surface analysis of silicon nanowires using capacitance-voltage measurements. Nat. Nanotechnol. 2009, 4, 311. 

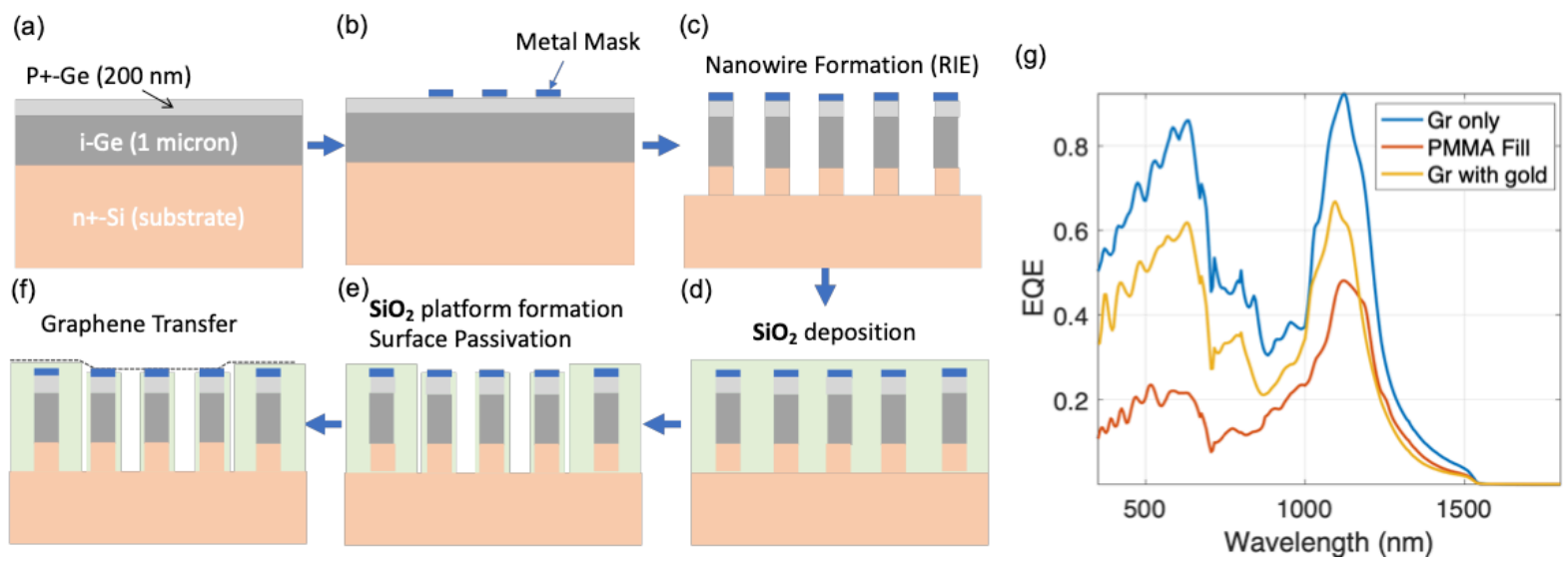

Figure 1. (a) - (f): Process flow for fabrication of Ge nanowire photodetectors with free-standing graphene top contact. (g) External quantum efficiency (EQE) vs wavelength, simulated for Ge nanowire photodetectors. Blue curve ("Gr only"): graphene electrode suspended on nanowires with no filling. Red curve ("PMMA fill”): graphene electrode on nanowires with PMMA filling. Orange curve ("Gr with gold"): top contact is graphene with thin gold layer ( $3 \mathrm{~nm}$ ) suspended on nanowires with no filling. Nanowires have radii of $90 \mathrm{~nm}$ and are in square arrays (period 800 $\mathrm{nm})$. Nanowires are $1500 \mathrm{~nm}$ tall, with top section being Ge $(1200 \mathrm{~nm})$ and bottom section being Si (300 nm). Substrate is Si. EQE represents fractional absorption in intrinsic region of Ge nanowire (200 nm from top and extending $1000 \mathrm{~nm}$ ). $\mathrm{SiO}_{2}$ coating is $80 \mathrm{~nm}$ thick. 


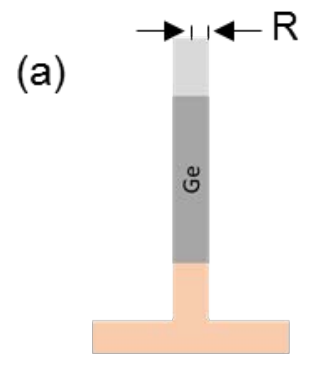

(d)

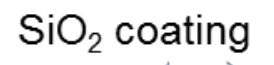

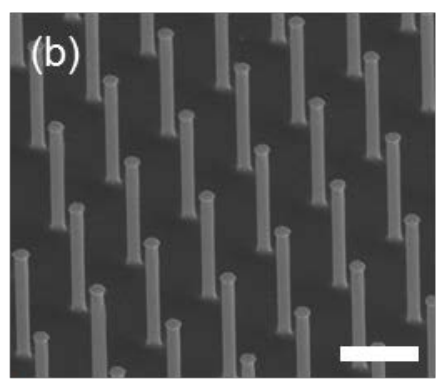

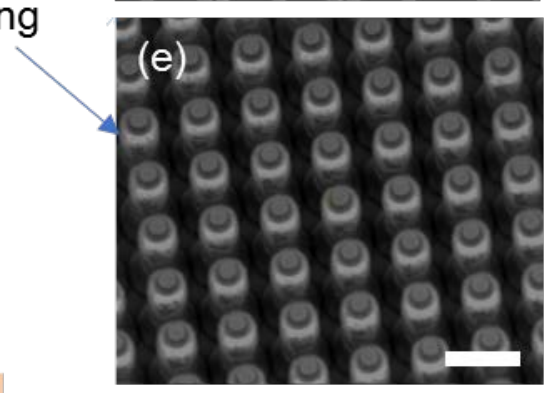

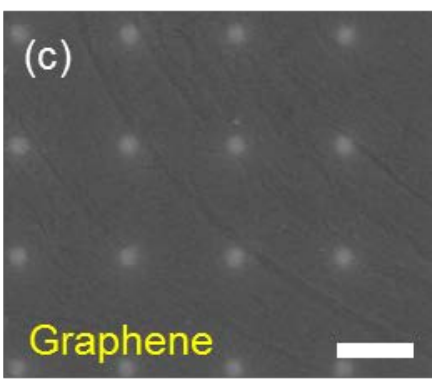

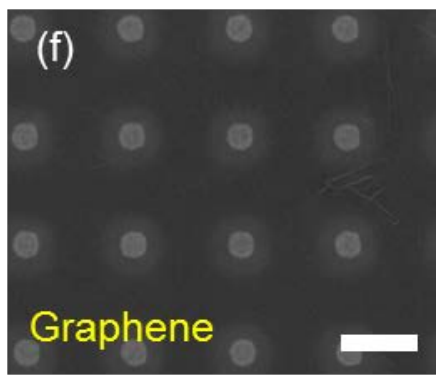

Figure 2. (a) Side-view schematic of NW-PD. (b) SEM image of NW-PD before graphene transfer (view tilted by 52 degrees). (c) Top-view SEM after graphene transfer. (d) Side-view schematic of p-NW-PD. (e) SEM image of p-NW-PD before graphene transfer (view tilted by 30 degrees); (f) Top view SEM of p-NW-PD after graphene transfer. Scale bars have length 1 micron. 


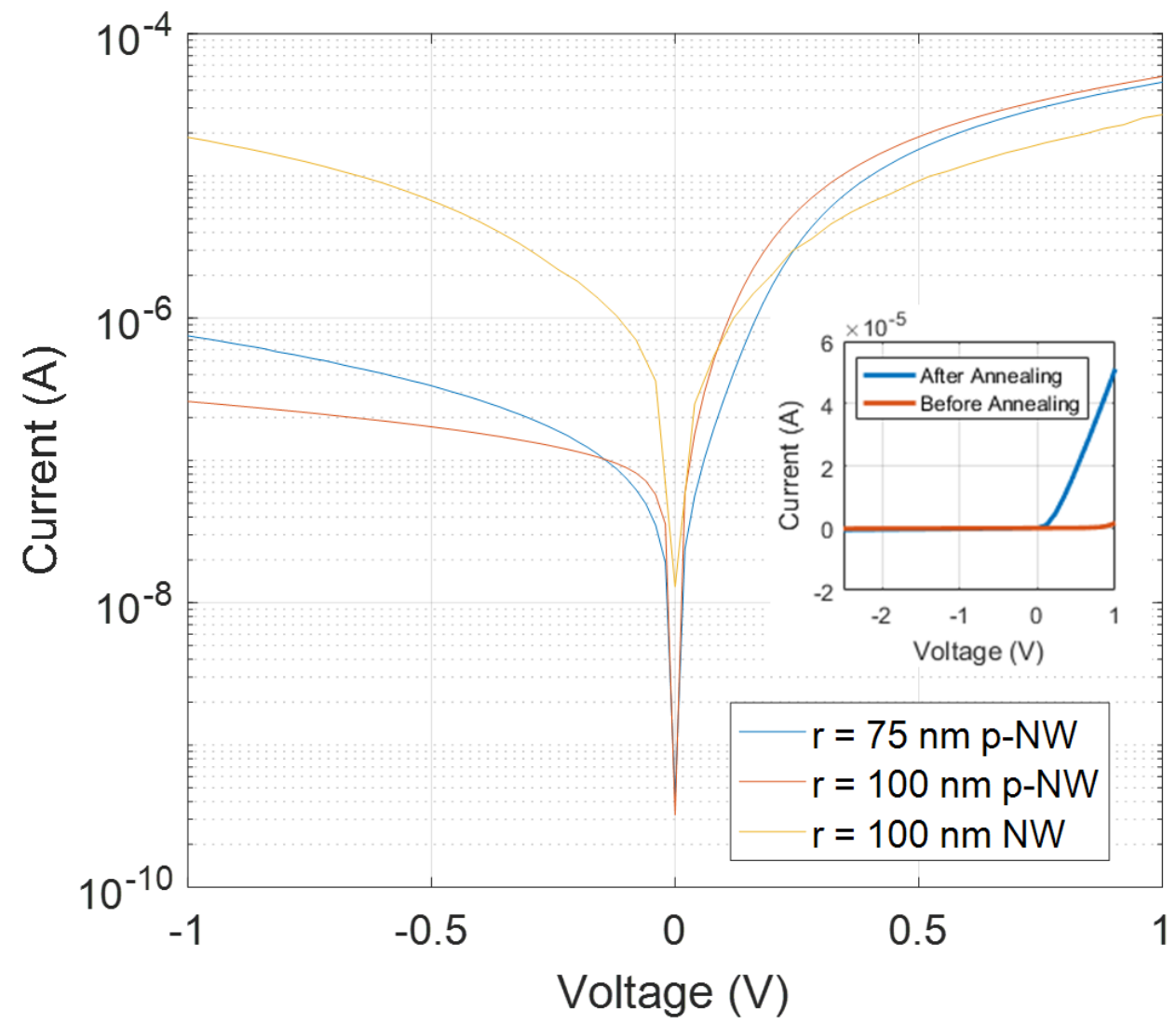

Figure 3. $I-V$ characteristics of nanowire photodetectors with (p-NW PD) and without passivation (NW PD). All three devices shown are final devices after annealing. Magnitude of current ( $|I|)$ is plotted. Insert: $I-V$ characteristics of passivated nanowire photodetectors $(\mathrm{r}=100 \mathrm{~nm})$ before and after annealing. 

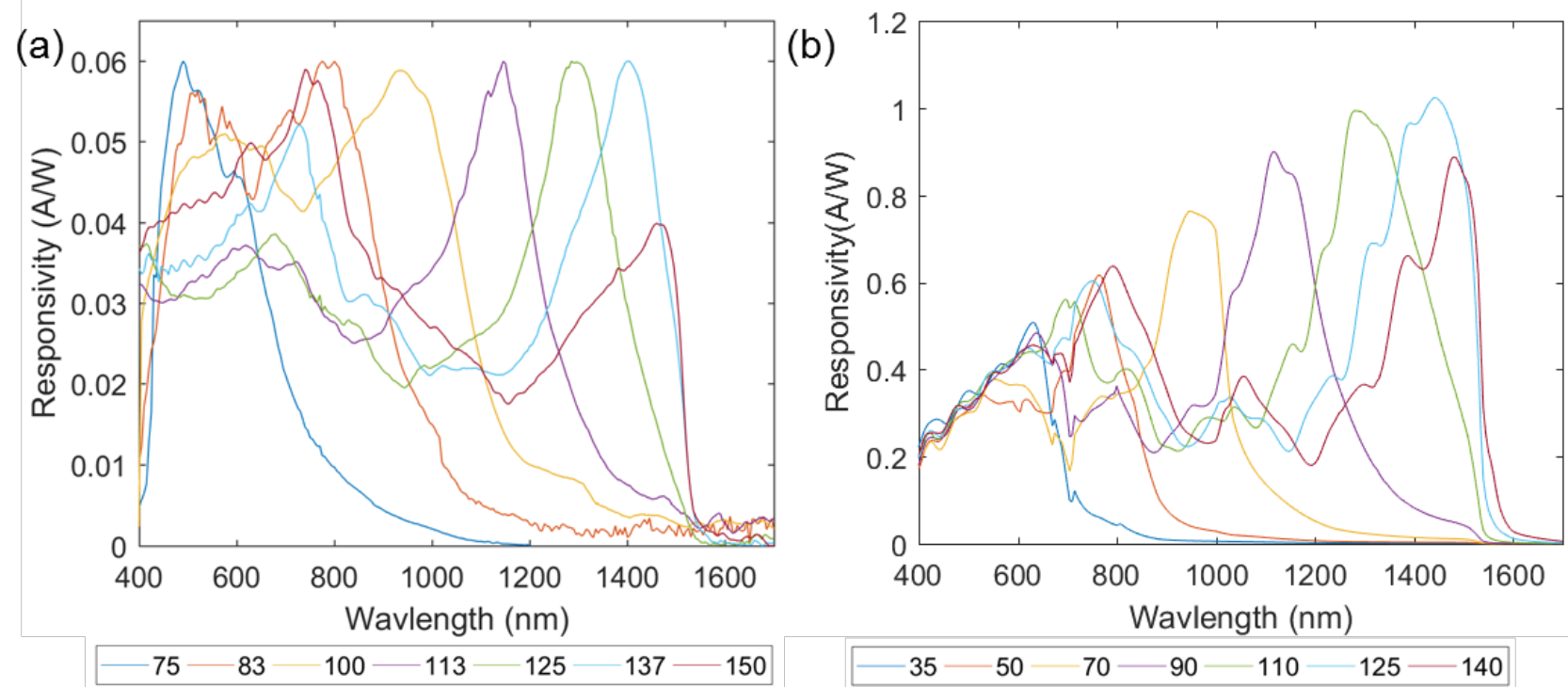

Figure 4(a). Measured responsivity spectra for p-NW-PD devices with different design radii. Due to large variation of responsivity, spectra are scaled for clarity of visualization. Scaling factors are $(13,300,22,180,12,1,25)$ times, in order of increasing nanowire radius. (b). Simulated responsivity spectra for $p$-NW-PD devices. Note that radii used in simulations are different to experimental design radii. 


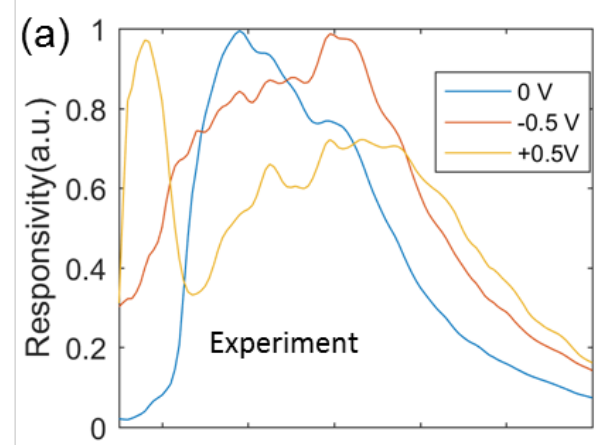

(c)
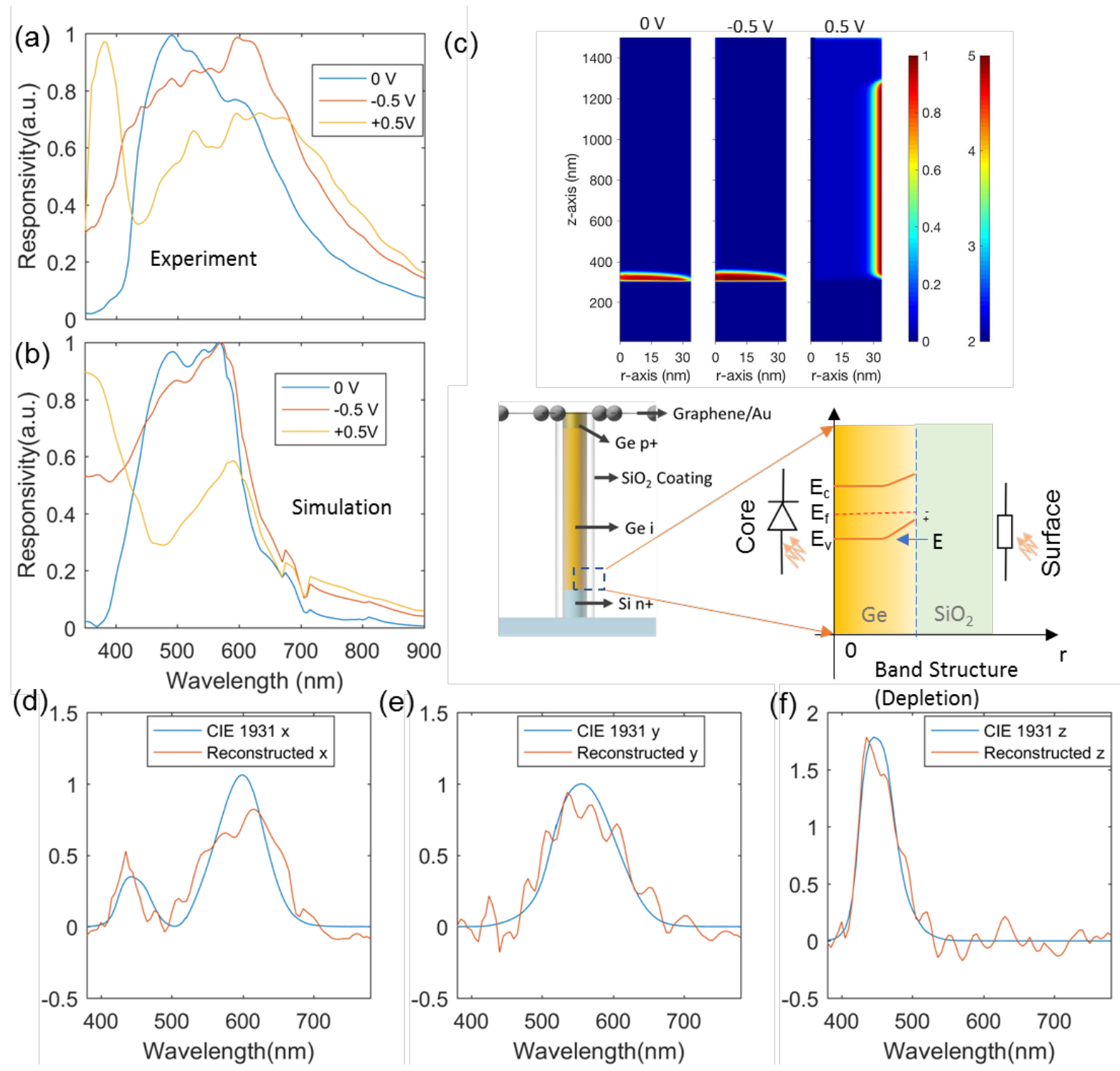

Figure 5. (a) Measured and simulated (b) responsivity spectra for p-NW-PD device at different bias voltages. (c). Upper panel: spatial IQE map at zero, reverse (-0.5 V) and forward bias (0.5 V). Vertical (z-axis) and horizontal (r-axis) axes of map indicate position along nanowire long axis and along radial direction, respectively. Left colorbar is for unbiased and reverse bias plots. Right colorbar is for forward bias. Bottom panel: schematic illustrations of nanowire device (left) and band diagram (right). (d)-(f). Original (blue curves) and reconstructed (orange curves) CIE 1931 observer $\mathrm{x}$, $\mathrm{y}$ and $\mathrm{z}$ photoresponses. Each reconstructed photoresponse comprises weighted sum of responsivity spectra measured from a single nanowire device (75 $\mathrm{nm}$ radius) under eight different voltages. Further details in Supplementary Information. 


\title{
For Table of Contents Use Only
}

\section{Vertical Ge-Si Nanowires with Suspended Graphene Top Contacts as Dynamically-tunable Multispectral Photodetectors}

\author{
Shi Qiang. Li ${ }^{1 \#}$, Amit Solanki ${ }^{2}$, Jacopo Frigerio ${ }^{3}$, Daniel Chrastina ${ }^{3}$, Giovanni Isella ${ }^{3}$, Changxi \\ Zheng ${ }^{4,5}$, Arman Ahnood ${ }^{6}$, Kumaravelu Ganesan ${ }^{6}$, and Kenneth B. Crozier ${ }^{1,6, *}$ \\ ${ }^{1}$ Department of Electrical and Electronic Engineering, University of Melbourne, Victoria 3010, Australia \\ ${ }^{2}$ School of Engineering and Applied Science, Harvard University, 33 Oxford Street, Cambridge, Massachusetts \\ 02138, United States \\ ${ }^{3}$ Dipartimento di Fisica del Politecnico di Milano, L-NESS, Polo Regionale di Como, Via Anzani 42, I-22100 \\ Como, Italy \\ ${ }^{4}$ School of Physics and Astronomy, Monash University, Clayton, Victoria 3800, Australia \\ ${ }^{5}$ ARC Centre of Excellence in Future Low-Energy Electronics Technologies, Monash University, Clayton, Victoria \\ 3800, Australia \\ ${ }^{6}$ School of Physics, University of Melbourne, Victoria 3010, Australia \\ *Corresponding author: Email kenneth.crozier@unimelb.edu.au \\ \#Current address: Institute of Materials Research and Engineering (IMRE), A*STAR, 2 Fusionopolis Way, \\ Singapore 138634
}

Synopsis: This TOC entry consists of an artistic rendering of our photodetector device, which consists of germanium-silicon nanowires with a graphene top contact.

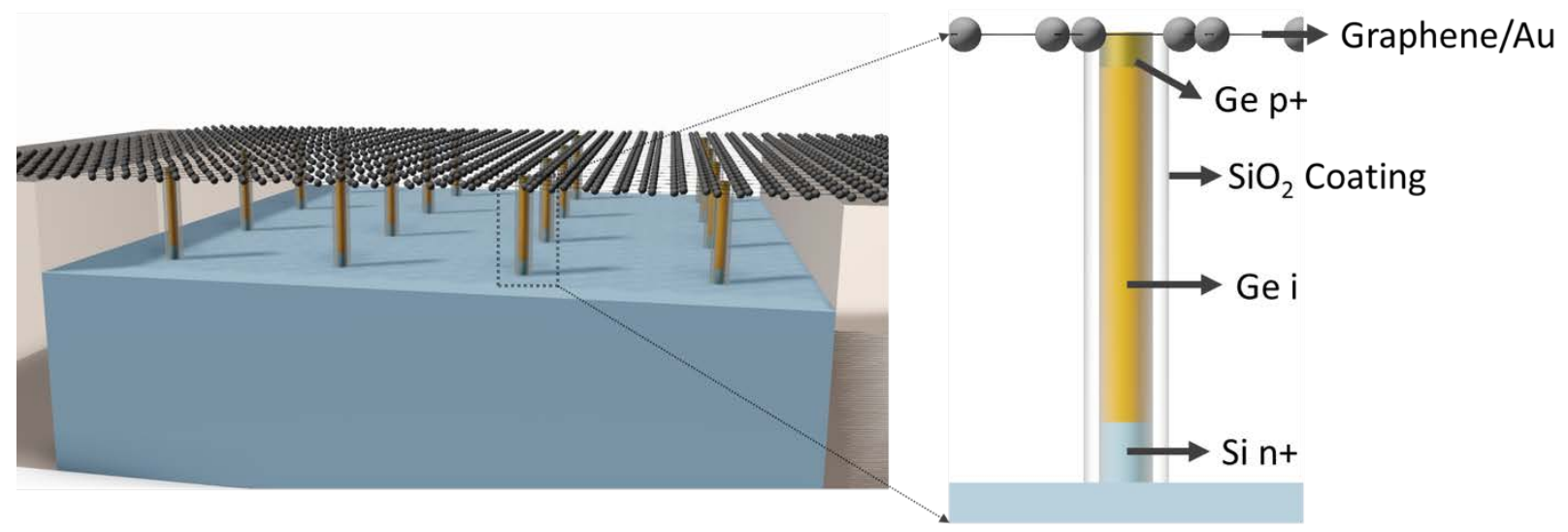

